\title{
SPACE LEAKAGE OF POWER FROM UNIPLANAR TRANSMISSION LINES
}

Jan Macháč, Ján Zehentner

\begin{abstract}
Surface and space leakage of power deteriorates the operation of circuits. On the other hand space leaky waves enable the design of specific radiators. In this paper we therefore investigate the potential of the slotline and the coplanar waveguide to radiate into space. We confirm the findings of earlier studies and extend them to higher order modes as regards the slotline, and report analogous new findings on the coplanar waveguide.
\end{abstract}

\section{Introduction}

In recent years we have investigated surface leaky waves on open uniplanar transmission lines. These waves, mostly undesirable, deteriorate the operation of circuits. On the other hand, leakage of power into space, usually also accompanied by surface leakage, is welcomed in antenna applications. We have therefore focused on this feature of open uniplanar transmission lines, represented in our choice by the slotline and the coplanar waveguide, cross-sections of which are shown in Fig. 1.

We used the method of moments in the spectral domain for analysing uniplanar transmission lines. The first task was to modify the integration path at the plane of the complex spectral variable when calculating integrals representing elements of the determinantal dispersion equation, the solution of which provides a propagation constant. To check our procedure we compared our own results with those published in [1] for a slotline with infinite-conductor planes. We computed the dispersion characteristics of the first higher bound mode denominated in [1] as the surface-wave-like mode, and the space leaky wave derived from this 1 st higher order bound mode. Since the differences between published and our characteristics were negligible, we applied this method in the analysis of all additional investigated lines.

In this paper we offer for a discussion new original solutions of the dispersion equation of the slotline, providing a modified view of constituent space leaky waves. We also introduce their correspondence with modes published for the slotline in [1] and for the suspended microstrip line in [2]. Additionally, we report theoretical identification of space leaky waves on the coplanar waveguide. This type of leaky wave was discovered and later experimentally verified on the suspended microstrip line [2]. A comparison of already known operation modes of the various open transmission lines that are discussed here confirms their common features and similarity of behaviour.

\section{Leakage of power from a slotline}

Firstly we feel the need to unify the terminology introduced by several authors dealing with the same phenomena. We classify and name waves according to the even and odd distribution of the transversal electric field component within the slot referring to a plane of symmetry placed perpendicular to the slot in its middle, and according to the number of changes of orientation of this field component within the slot. Accordingly, the dominant bound wave does not change orientation and has even symmetry of the electric field component with respect to the electric wall placed in the position of the plane of symmetry. When the electric field component has odd symmetry with respect to the magnetic wall located in the plane of symmetry and changes its orientation once we have the 1st higher order bound wave. Next is the 2nd higher order bound wave with even symmetry and two changes of field orientation. The 3rd higher order bound wave exhibits odd symmetry of the electric field component, which changes orientation three times, etc. In other words, we have even modes $\mathrm{E}_{0}, \mathrm{E}_{2}, \mathrm{E}_{4}, \ldots$ and odd modes $\mathrm{O}_{1}, \mathrm{O}_{3}, \mathrm{O}_{5} \ldots . \mathrm{E}_{0}$ is the dominant bound mode. With rising frequency, they form a sequence $\mathrm{E}_{0}, \mathrm{O}_{1}$, $\mathrm{E}_{2}, \mathrm{O}_{3}, \mathrm{E}_{4}, \ldots$ where the subscript denotes the number of changes of field orientation. We therefore speak about the dominant and 1st, 2nd, 3rd, 4th, etc., bound wave, i. e., about the dominant and higher order modes. Consequently our 1st higher order bound wave is denominanted in [1] as the surface-wave-like mode.

Space leaky waves can be divided into two groups. The first group contains waves leaking power into space and related to the higher order bound wave. Consequently there are the 1 st, 2nd, 3rd, 4th, etc., space leaky waves related to the sequence of the 1st, 2nd, 3rd, 4th, etc., higher order bound waves. For historical reasons they were denoted as $\mathrm{EH}_{1}, \mathrm{EH}_{2}, \mathrm{EH}_{3}$, etc., modes, e. g., on the microstrip line [3]. The first leaky mode of this group on the slotline, denominated the first higher order mode, is discussed in [1]. Occurrence of higher order bound and

Czech Technical University, Technická 2, 16627 Prague 6, Czech Republic, Tel +420 224352273, Fax +420 2

24355865, E-mail zeh@mbox.cesnet.cz 
space leaky waves is typical for wide slots. The second leaky wave group contains so called space-wave leaky modes carrying dominant-mode-like currents [2,3]. In the case of the slotline, their transverse electric field components have a distribution very similar to those of the corresponding bound wave. This family of space leaky waves was first revealed on the suspended microstrip line [2].

The dispersion characteristics in Fig. 2 provide a compact representation of findings relevant to the slotline. Firstly, there is the normalized phase constant of the 1st and 3rd higher order bound wave. The existence of this solution of the dispersion equation appeared for the 1st higher order bound wave in [1] and we checked the published data by means of our code. A solution for the 3rd higher order bound wave was reported in [4]. Secondly, the normalized phase and leakage constants of the 1st space leaky wave, published in [1], were also checked by our code. This demonstrates the correctness of our way of computation. This wave belongs to the first group of space leaky waves introduced above. In Fig. 2 we denoted this as the 1 st space leaky wave of class 1 , i. e. , $1 \mathrm{stSL}_{\mathrm{Cl}}$. Distribution of its transversal component of the electric field $\mathrm{E}_{\mathrm{x}}$ within the slot and of the 1st higher order bound wave are plotted in Fig. 3. Thirdly, Fig. 2 shows new normalized phase and leakage constants of the 1 st space leaky wave of class 2 , labeled $1 \mathrm{stSL}_{\mathrm{C} 2}$. Its component of $\mathrm{E}_{\mathrm{x}}$ is drawn in Fig. 3. $1 \mathrm{stSL} \mathrm{C}_{\mathrm{C} 1}$ and $1 \mathrm{stSL}_{\mathrm{C} 2}$ waves are physical when their $\beta$ are lower than $\mathrm{k}_{0}$. However, a high value of $\alpha$ does not directly predict their high excitation efficiency. $\mathrm{E}_{\mathrm{x}}$ of the $1 \mathrm{stSL}_{\mathrm{C} 1}$ and $1 \mathrm{stSL}_{\mathrm{C} 2}$ wave resemble one another and are also both as $\mathrm{E}_{\mathrm{x}}$ of the $1 \mathrm{st}$ higher order bound wave.

Two new findings indicating the potential of the slotline to leak power into space are shown in Fig. 4 . The 2 nd higher order bound wave is physical when its $\beta$ is greater than $\mathrm{k}_{\mathrm{TM} 0}$. With decreasing frequency it continuously passes into an improper nonphysical solution. This, for selected substrate permittivity, decreases in the narrow frequency band below $\mathrm{k}_{0}$ and represents a physical space leaky wave. When the frequency falls further and $\beta$ is again greater than $\mathrm{k}_{0}$ we get an improper complex solution, which is nonphysical. This solution corresponds to the 2 nd space leaky wave of class 1 , i. e., briefly $2 \mathrm{ndSL}_{\mathrm{C} 1}$. Note that it has an upper cutoff frequency when $\alpha$ falls to zero. Additionally there is also the 2 nd space leaky wave of class 2, the dispersive characteristics of which are denoted $2 \mathrm{ndSL}_{\mathrm{C} 2}$. This wave is physical so long as its $\beta$ is below $\mathrm{k}_{0}$ and has a wide frequency range. The distribution of the magnitude and phase of $\mathrm{E}_{\mathrm{x}}$ of the $2 \mathrm{ndSL}_{\mathrm{C} 1}$ and $2 \mathrm{ndSL}_{\mathrm{C} 2}$ within the slot are plotted, for the slotline from Fig. 4, in Fig. 5 along with $E_{x}$ of the 2nd higher order bound wave. It can be seen that $E_{x}$ of all three waves resemble one another. So far these two types of space leaky waves have been observed only in conjunction with modes having odd symmetry of $\mathrm{E}_{\mathrm{x}}$.

\section{Leakage of power from a coplanar waveguide}

Analysis of a coplanar waveguide in the source-free region provides particular components of the total field propagating over the line. We will now deal only with waves having odd symmetry of $E_{x}$ with regard to the central plane of symmetry of the line lying perpendicularly in the middle of the inner strip conductor. Distribution of $E_{x}$ within one slot is now either semi-even or semi-odd in consequence of a shift of the slot aside the longitudinal axis of the line. In this sense, $\mathrm{E}_{\mathrm{x}}$ of the dominant bound wave again has a semi-even symmetry with regard to the plane of symmetry located in the middle of the slot, while the 1st higher order bound wave has semi-odd symmetry. When we search for space leaky waves the dispersion equation extends new characteristics which, after normalization to the wave number of the free space $\mathrm{k}_{0}$, are plotted in Fig. 6 . All curves were computed with the same number of basis functions and are related to the 1 st higher order bound wave on the coplanar waveguide. A number of branches were subsequently found along the frequency axis, but only five of them are plotted here. They correspond to the simultaneous space and surface leakage, since the branch cut and the pole corresponding to the $\mathrm{TM}_{0}$ surface wave were taken into account in their calculation. We believe that waves with the characteristics introduced here are analogous to a family of waves revealed on the suspended microstrip line [2]. Their transversal electric component $\mathrm{E}_{\mathrm{x}}$ within the slot of the coplanar waveguide resembles that of the 1st higher order bound wave, Fig. 7.

\section{Conclusions}

From the dispersion equation in the source-free region we get a spectrum of the propagation constants of the constituent independent waves which may exist on the investigated line. This is also the target of this paper. However, the waves that are actually excited depend on the source, the feeding structure and the frequency. The source stimulates excitation of some waves and suppresses the rest of them.

Detailed browsing in solutions of the dispersion equations of the slotline and the coplanar waveguide has revealed new, so far unpublished modes representing space leakage. We have identified on the slotline the 1st space leaky wave of the second class, which has a much wider frequency band than the already known space leaky wave of the first class. There is not only leakage from modes with odd symmetrry of the electric field on the slotline but there are also modes with even symmetry that can radiate. We have documented this by means of 
the 2 nd space leaky wave of class 1 and class 2 . The $2 \mathrm{ndSL}_{\mathrm{C} 1}$ mode has a very narrow frequency band depending on the substrate permittivity. Moreover, it possesses the upper cutoff frequency. The frequency range for the $2 \mathrm{ndSL}_{\mathrm{C} 2}$ is much wider. We have shown that the coplanar waveguide is also able to leak power into space in the form of the $1 \mathrm{stSL} L_{\mathrm{C} 2}$.

We presume that all waves identified and quoted above are a part of the total field excited by a real source which in a different approach [5] is called a „residual wave“. Nevertheless there are several features typical of a few open transmission lines which can be considered general. The spectrum of bound and leaky waves provides an example.

\section{Acknowledgments}

This work has been supported by the Grant Agency of the Czech Republic under project „Planar circuits for mw and mmw technology“ 102/00/0025 and by the research programme J04/98 212300014 „Information and Communication Technology", and was carried out on the SP-2 computer at the Joint Supercomputer Center of the Czech Technical University, University of Chemical Technology and IBM Prague.

\section{References}

[1] J.-W. Sheen, Y.-D. Lin: Propagation Characteristics of the Slotline First Higher Order Mode. IEEE Trans. Microwave Theory Tech., vol. MTT-46, pp. 1774-1781, Nov. 1998.

[2] C.-K. C. Tzuang, C.-C. Lin: Fast-Wave Resonance of Space-Wave Leaky Mode Carrying Dominant-ModeLike Currents. IEEE Trans. Microwave Theory Tech., vol. MTT-46, pp. 2444-2449, Dec. 1998.

[3] C.-K. C. Tzuang: Leaky Mode Perspective of Printed Antenna. 1998 Asia-Pacific Microwave Conference Proceedings, vol. 3, pp. 1471-1478, 1998 Yokohama, Japan.

[4] K. Mejsnar: Higher-Order Modes on Slot Lines. 10th European Microwave Conference Proceedings, pp. 327-330, 1980 Warszawa, Poland

[5] M. J. Freire, F. Mesa, D. R. Jackson: The Residual-Wave Current Excited on a Printed-Circuit Line: A Source of Spurious Effects. 29th European Microwave Conference Proceedings, vol. 3, pp. 114-117, 1999 Munich, Germany.

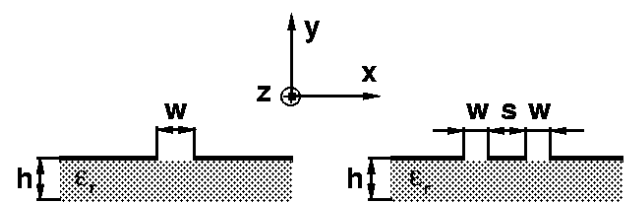

Fig. 1 Cross-section of the slotline and the coplanar waveguide.

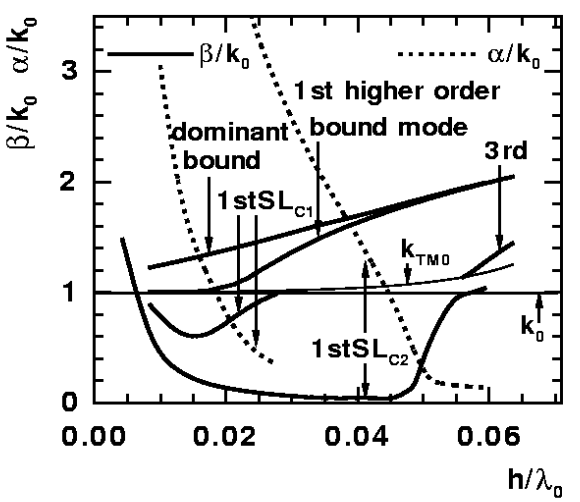

Fig. 2 Dispersion characteristics of the dominant, the 1 st and 3rd higher order bound waves, and of the $1 \mathrm{st}$ space leaky waves of class 1 and class 2 on the slotline with $\varepsilon_{\mathrm{r}}=10.8, \mathrm{w}=20 \mathrm{~mm}, \mathrm{~h}=1.27 \mathrm{~mm}$.

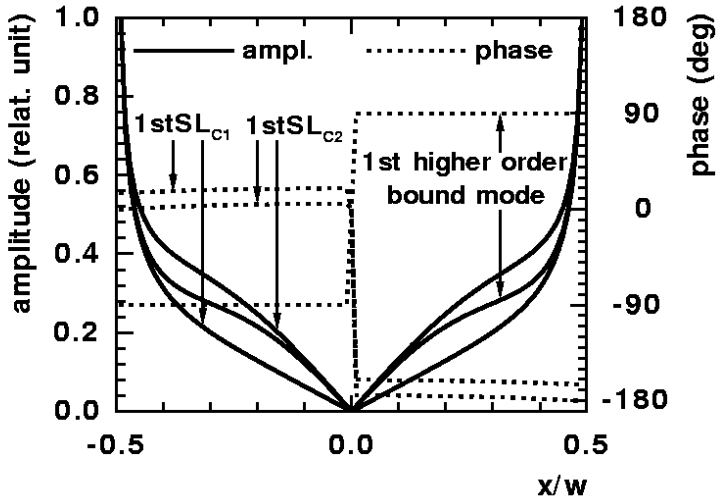

Fig. 3 Distribution of amplitude and phase of the transversal component of the electric field within the slot for the 1 st space leaky wave of class 1 and 2 , and for the 1st higher order bound wave on the slotline from Fig. 2 at $\mathrm{h} / \lambda_{0}=0.0212$. 


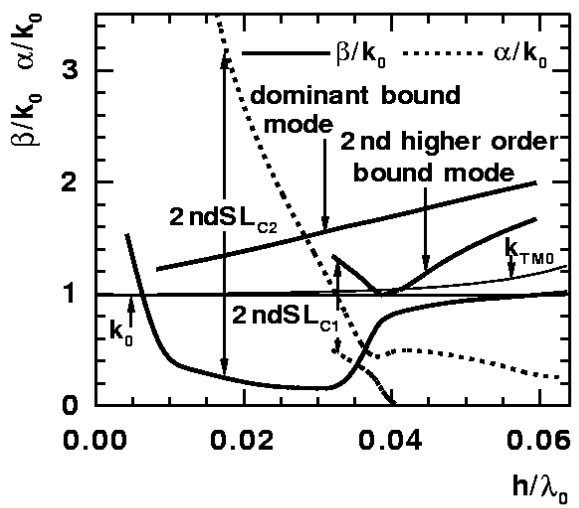

Fig. 4 Dispersion characteristics of the dominant, the 2 nd higher order bound wave, and the 2 nd space leaky waves of class 1 and class 2 on the slotline with $\varepsilon_{\mathrm{r}}=10.8, \mathrm{w}=20 \mathrm{~mm}, \mathrm{~h}=1.27 \mathrm{~mm}$.

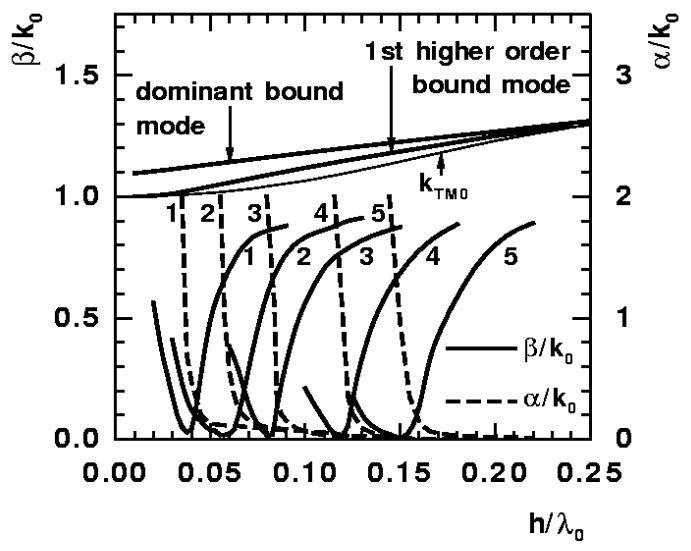

Fig. 6 Dispersion characteristics of the space leaky waves on the coplanar waveguide with $\varepsilon_{\mathrm{r}}=2.25, \mathrm{w}=20$ $\mathrm{mm}, \mathrm{s}=1 \mathrm{~mm}, \mathrm{~h}=1 \mathrm{~mm}$.

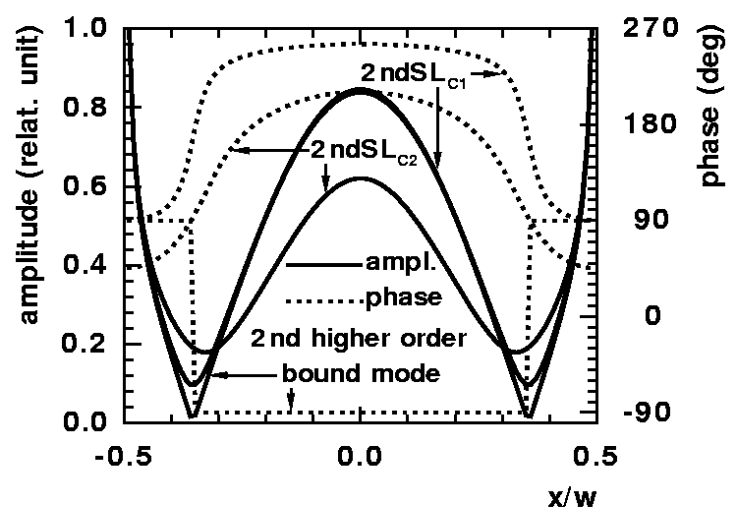

Fig. 5 Distribution of amplitude and phase of the transversal component of the electric field within the slot for the 2nd space leaky wave of class 1 and 2 at $\mathrm{h} / \lambda_{0}=0.0389$, and for the $2 \mathrm{nd}$ higher order bound wave on the slotline from Fig. 4 at $\mathrm{h} / \lambda_{0}=0.0444$.

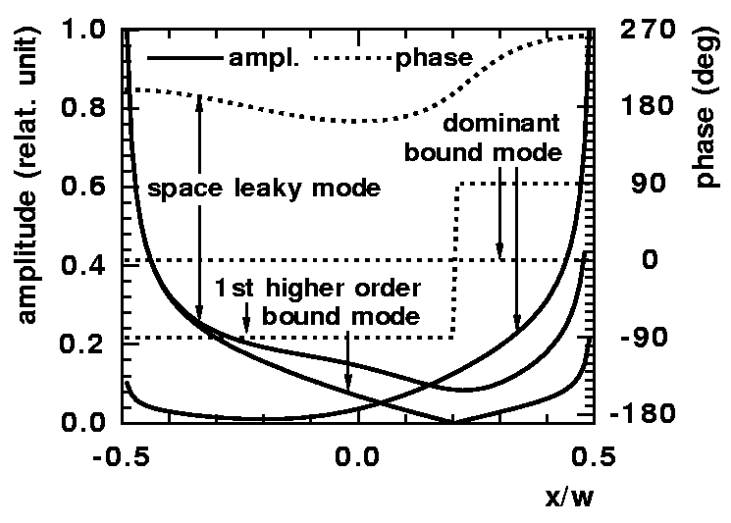

Fig. 7 Distribution of amplitude and phase of the transversal component of the electric field within the left slot of the coplanar waveguide from Fig. 6 for the dominant and the 1st higher order bound waves and for the first branch of the space leaky wave from

Fig. 6 when $\mathrm{h} / \lambda_{0}=0.055$. 